\title{
EXPERIMENTAL VALIDATION FOR AN EXTENDED-STABILITY ELECTROSTATIC ACTUATOR
}

\author{
S. Towfighian \\ Mechanical Engineering \\ University of Waterloo \\ Waterloo, ON N2L 3G1, Canada \\ Email: stowfigh@engmail.uwaterloo.ca \\ E. M. Abdel-Rahman* \\ Systems Design Engineering \\ University of Waterloo \\ Waterloo, ON N2L 3G1, Canada \\ Email: eihab@engmail.uwaterloo.ca
}

\author{
A. Seleim \\ Systems Design Engineering \\ University of Waterloo \\ Waterloo, ON N2L 3G1, Canada \\ Email:asseleim@uwaterloo.ca \\ G. R. Heppler \\ Systems Design Engineering \\ University of Waterloo \\ Waterloo, ON N2L 3G1, Canada \\ Email: heppler@uwaterloo.ca
}

\section{ABSTRACT}

A voltage regulator is developed to extend the operation range of a electrostatic actuator using a displacement feedback. The feedback actuator system can be used for continuous position tracking of step, ramp, or harmonic voltage signals. The electrostatic actuator is composed of a micro-cantilever beam electrode above a fixed electrode. The voltage difference between the two electrodes is regulated by the controller to maintain the balance between mechanical and electrostatic forces at large beam deflections, thereby increasing the actuation range. Simulated closed loop system responses with experimentally identified parameters are presented and show the actuation can reach up to $85 \%$ of the gap in the static response with an input voltage of less than $12 \mathrm{~V}$. Experimental results show that the controller properly functions and prevents the beam from experiencing pull-in. A close agreement is found between the simulated

*Address all correspondence to this author. and measured closed loop model dynamic responses.

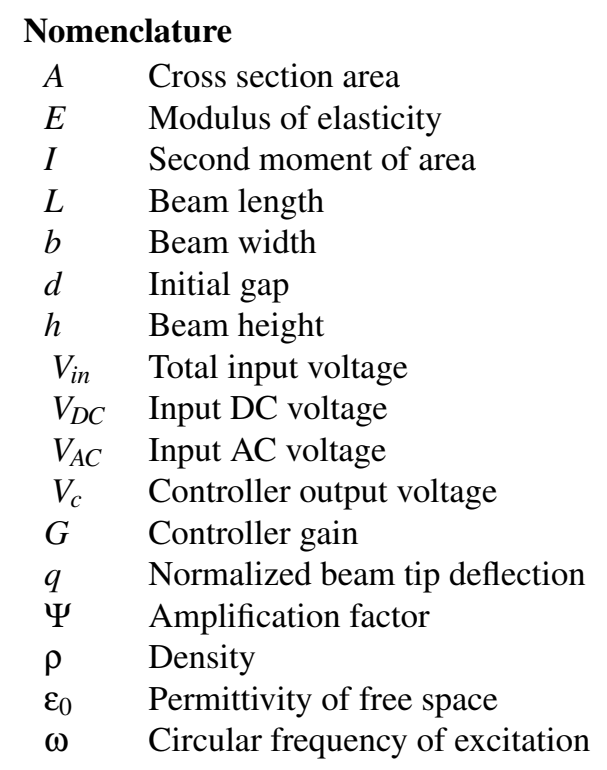




\section{Introduction}

MEMS actuators have been widely used in many fields, including micro-manipulation [1-3], micro-assembly [4, 5], biomedical applications [6,7] and probe-based magnetic microdisc drives [8]. MEMS acatuaros are divided into four groups including electrostatic, thermal, piezoelectric and magnetic. Electrostatic actuation has the advantage of ease of fabrication and integration with CMOS technology in addition to energy efficiency compared to other actuation types $[9,10]$.

In electrostatic actuation, two electrodes move against each other under the influence of an electrostatic force that arises due to a voltage difference between the electrodes. The major drawback of electrostatic actuation is the narrow stable operating region, which is only $0.3-0.4$ of the gap between the two plates. Inside this stable region the position of the moving plate is determined by the balance between the electrostatic and mechanical forces. Beyond 0.3-0.4 of the gap, the mechanical force is no longer capable of balancing the nonlinear electrostaic force and pull-in takes place [11]. Several approches have been proposed to overcome this drawback including leveraged bending where plates are operated in the stable region and leverage is used to increase the travel [10], charge control where capacitance is added in series with the actuator to overcome the pull-in instablity [12], and feedback control [13-16]. Feedback control has more advantages over the other methods. It can stabilize the system beyond the pull-in instability in addition to adding robustness to the system against parameter uncertainties and deterioration over time [17].

Feedback control was first suggested by Chu and Pister in [13]. They used a nonlinear controller in which the input voltage is scaled by the gap. In the simulations, they were able to produce a travel range larger than $80 \%$ of the gap. Lu and Fedder [14] then implemented a position feedback using analog electronics and a capacitor as a position sensor. They used a linear time-invariant controller to simplify implementation and linearized the system while using gain and phase margin to ensure stability. They were able to obtain a stable travel range of $60 \%$ of the gap in the experiments. Rocha et al. [15] achieved over $90 \%$ of stable travel in experiment using on-off control with capacitive position sensing. A comparator is set to compare the position of the beam with a set position, as long as the position of the beam is lower than the set position, the input voltage is higher than the pull-in voltage. When the beam position is larger than the set position, the input voltage switches to a voltage lower than the pull-in voltage. The beam keeps oscillating around the required position and the ripples magnitude depend on the delays in the controller circuit. The ripples magnitude can be very high and cause pull-in in systems with high Q factor. Vagia et al. [16] used a model of a moving plate and where able to achieve $3.9 \mu \mathrm{m}$ travel in a $4 \mu \mathrm{m}$ gap device in simulations using a PID controller.

In this paper, experimental verification is presented for a feedback control approach presented by Lu et al. [14] and further developed in references $[18,19]$. This controller is designed for an electrostatically actuated micro-cantilever beam. The microbeam is mounted on a vibrometer that is used as a sensor, yielding a voltage signal representing the velocity of the tip of a microbeam. An analog electronic circuit is then used to realize the controller and close the loop with the micro beam. After validating the closed-loop system dynamics, the vibrometer can be replaced by a piezoresistor to sense the position, thus eliminating the parasitic behavior due to the presence of capacitors [14].

In the sequel the mathematical modeling of the system, parameter identification, and static and dynamic responses of the closed loop system are illustrated from both simulations and experiments. A discussion on the results, and conclusions concludes the paper.

\section{System Model}

The open loop system is an electrostatic actuator consisiting of a fixed electrode with a moving electrode above it (Figure 1). The moving electrode is a micro-cantilever beam that is driven by applying different voltages between the two electrodes.

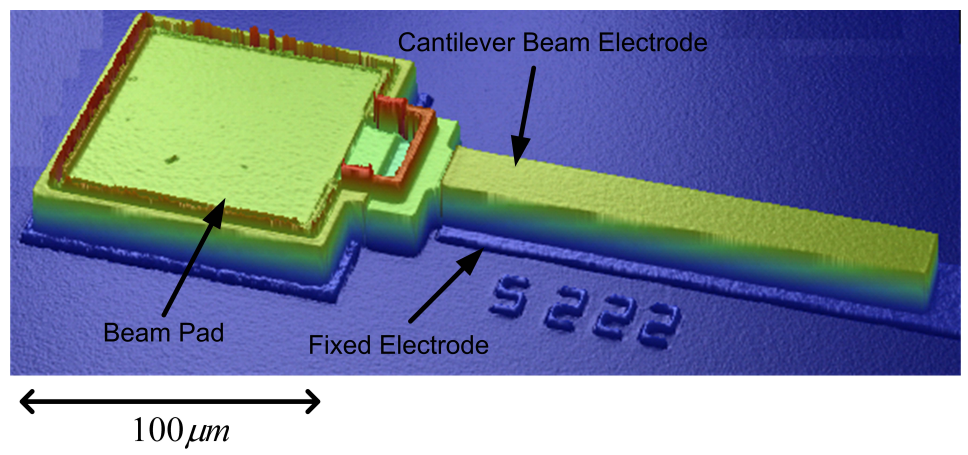

Figure 1: The electrostatic actuator under testing. (picture is taken by an optical profilometer)

Using the Euler-Bernoulli beam model and following Nayfeh et al. [20] for reduced order models, an open loop model is obtained employing a one-mode approximation in Galerkin's method (for more details, see [21]) as

$$
\left(\ddot{q}+\mu \dot{q}+\omega_{1}^{2} q\right)\left(1+c_{1} q+c_{2} q^{2}\right)=c_{3} \alpha V_{i n}^{2}
$$

where $q$ is the non-dimensional micro-cantilever beam tip deflection (nondimensionalized with respect to the initial gap) and time is nondimensionalized with respect to

$$
T=\sqrt{\frac{\rho A L^{4}}{E I}}
$$


In Equation 1, overdot means derivative with respect to time, $\omega_{1}$ is the first canonical natural frequency of the beam, $V_{i n}$ is the input voltage to the system given by

$$
V_{\text {in }}=V_{D C}+V_{A C} \cos (\omega t)
$$

and $c_{1}, c_{2}$, and $c_{3}$ are found after applying Galerkin's method. The two introduced parameters are

$$
\mu=2 \zeta \omega_{1}, \alpha=\frac{\varepsilon_{0} b L^{4}}{2 E I d^{3}}
$$

where $\alpha$ is the electromechanical coupling coefficient, $\zeta$ is the damping ratio determined experimentally, and the other parameters are defined in Table 1.

Table 1: The electrostatic actuator parameters

\begin{tabular}{|l|c|c|}
\hline Parameter & Symbol & Value \\
\hline \hline Density & $\rho$ & $2331 \mathrm{~kg} / \mathrm{m}^{3}$ \\
\hline Damping ratio & $\zeta$ & 0.08 \\
\hline Permittivity of air & $\varepsilon$ & $8.85 \times 10^{-12} \mathrm{~F} / \mathrm{m}$ \\
\hline Modulus of Elasticity & $E$ & $150 \mathrm{GPa}$ \\
\hline Amplification factor & $\Psi$ & $1 \mathrm{~V}$ \\
\hline Controller gain & $G$ & 2 \\
\hline
\end{tabular}

The closed loop feedback system is comprised of the mentioned electrostatic actuator and an electronic circuit controller, which regulates the voltage applied to the beam. The closed loop system is represented by an ordinary-differential equation:

$$
\left\{\begin{array}{l}
\left(\ddot{q}+\mu \dot{q}+\omega_{1}^{2} q\right)\left(1+c_{1} q+c_{2} q^{2}\right)=c_{3} \alpha G^{2}\left(V_{i n}-V_{c}\right)^{2} \\
V_{c}=\frac{q}{1-q} \Psi
\end{array}\right.
$$

where $\psi$ is the amplification factor [21].

The controller acts as a voltage regulator to prevent pullin. In the open loop system, as the beam bends, the mechanical spring force balances the electrostatic force up to $30-40 \%$ of the gap, beyond that electrostatic force dominates and pullin happens. In the closed loop system, as the beam deflection increases, the controller voltage $V_{c}$ increases, see Figure 2, and thereby the net actuation voltage, $G\left(V_{i n}-V_{c}\right)$, decreases. As a result the electrostatic forcing drops at large deflections helping the spring force balance the beam, and extending the actuator stable operating range.

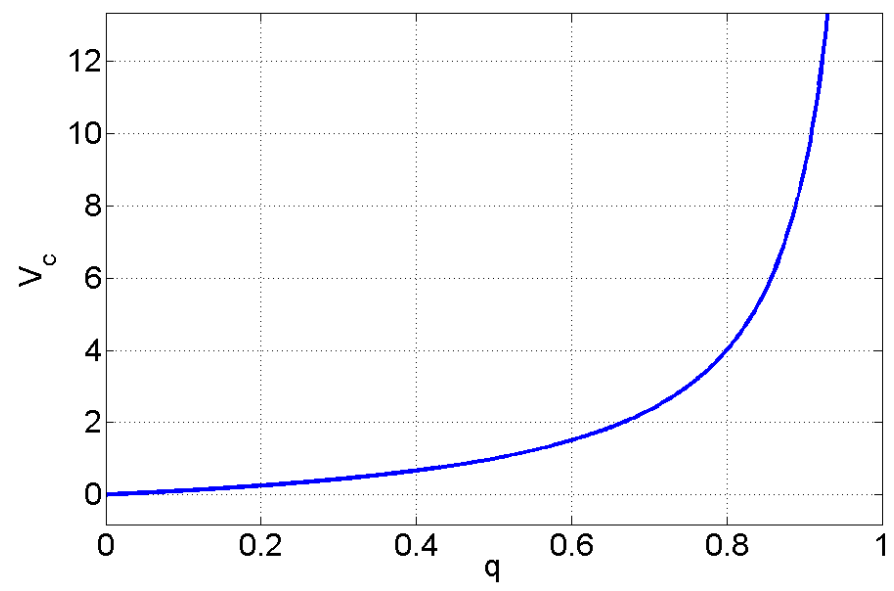

Figure 2: Controller voltage as a function of non-dimensional beam tip deflection: $V_{c}=\frac{q}{1-q}(\Psi=1 \mathrm{~V})$.

\section{Parameter Identification}

To identify the model parameters, the open loop system is tested to find the frequency responses and pull-in voltages. The frequency response curves of the electrostatic actuator are obtained experimentally and used to identify the beam length, pullin voltage, and damping ratio. Thickness and gap for the beams are measured using an optical profilometer, Figure 1.

Figure 3 illustrates experimental frequency response curves of the beam tip velocity at $V_{A C}=2 \mathrm{~V}$ and different DC voltages. In Figure 3, it is noted that the peak frequency decreases with increasing DC voltage. This is shown in Figure 4 that shows the natural frequency of the cantilever beam as a function of DC voltage from the eigenvalue calculations and from the experimental frequency response. One can identify the beam length by determining the natural frequency at $V_{D C}=0$, and the pull-in voltage by determining the voltage at the zero natural frequency. The natural frequency at $V_{D C}=0$ is found to be $103.3 \mathrm{kHz}$, and hence the effective beam length is determined to be $166.1 \mu \mathrm{m}$. Pull-in voltage is predicted to be $23.5 \mathrm{~V}$ from the eigenvalue calculation in the simulations.

From the frequency responses in Figure 3, the damping ratio is obtained by finding the compliance frequency response curves (beam tip velocities divided by individual frequencies), and calculating

$$
\zeta=\frac{\omega_{a}-\omega_{b}}{2 \omega_{p}}
$$


where $\omega_{a}, \omega_{b}$ are the frequencies where the magnitude is $1 / \sqrt{2}$ of the peak magnitude, and $\omega_{p}$ is the frequency of the peak. Damping ratio is calculated and averaged for the frequency response curves at $V_{D C}=6 \mathrm{~V}$, and $V_{D C}=11.2 \mathrm{~V}$ and is found to be 0.08 .

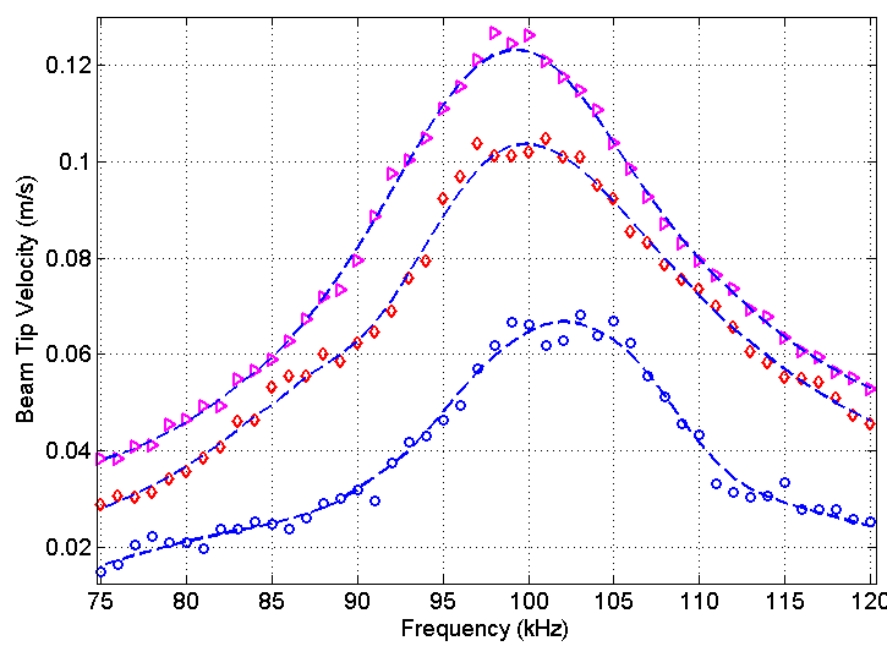

Figure 3: Frequency response of the beam tip velocity $(b=10 \mu m, h=$ $2.2 \mu m, d=2.1 \mu m$ ) at $V_{A C}=2 \mathrm{~V}: \circ V_{D C}=6 \mathrm{~V}, \diamond V_{D C}=9.07 \mathrm{~V}$, $\triangleright V_{D C}=11.2 \mathrm{~V},--$ fitted data on the experimental results.

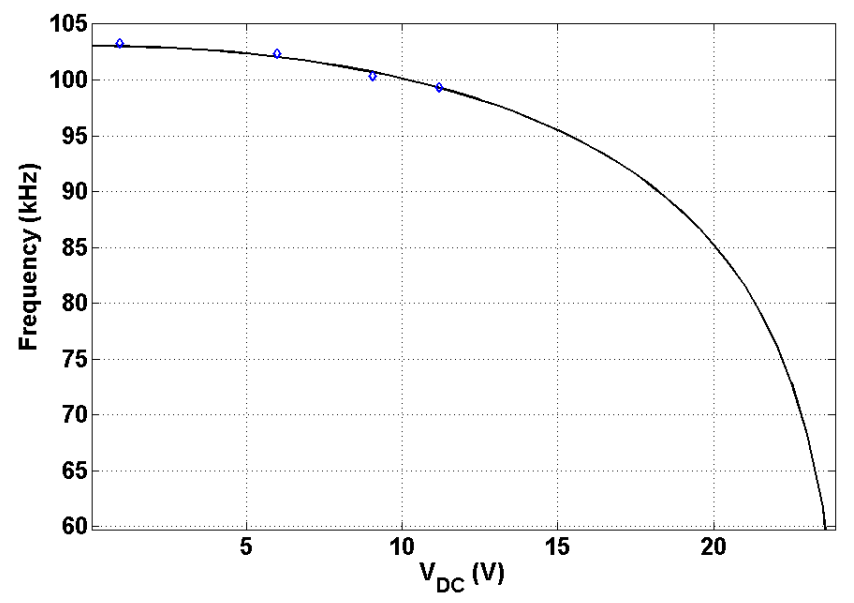

Figure 4: Natural frequency as a function of DC voltage for the beam with dimensions of $L=166.1 \mu \mathrm{m}, b=10 \mu \mathrm{m}, h=2.2 \mu \mathrm{m}$, and $d=$ $2.1 \mu m:-$ Simulations, $\diamond$ experimental results.

Using the beam length and damping ratio determined experimentally, Equation 1 is solved numerically. The simulation result is shown by a solid line in Figure 5. The figure shows that the one-mode model with the experimentally identified parameters yields a good approximation of the open loop dynamic behavior.

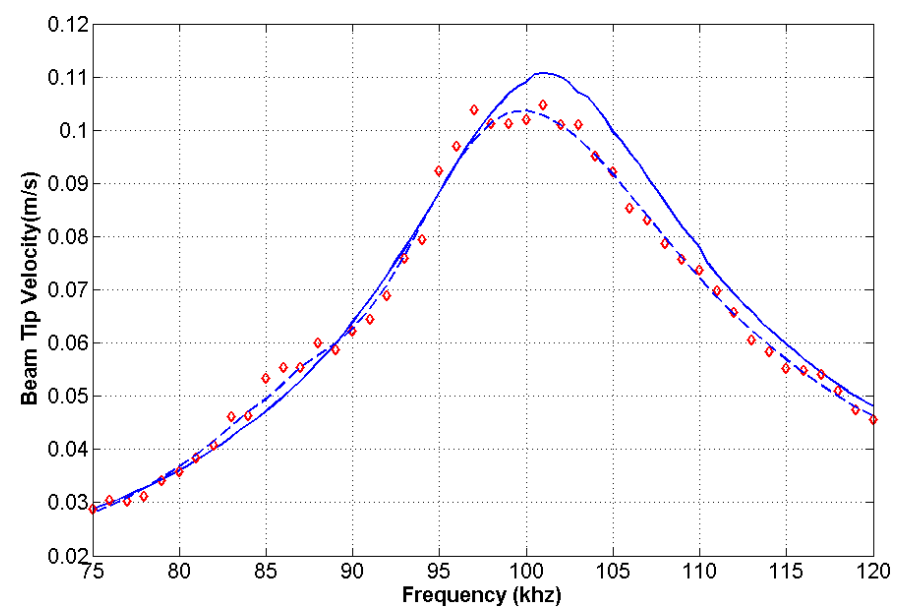

Figure 5: Frequency response of the beam tip velocity with dimensions of $L=166.1 \mu m, b=10 \mu m, h=2.2 \mu m, d=2.1 \mu m$ at $V_{D C}=9.07 \mathrm{~V}$, and $V_{A C}=2 \mathrm{~V}:-$ Simulations, $\diamond$ experimental results, -- fitted data on the experimental results.

For the closed loop test, a beam with the nominal length of $175 \mu \mathrm{m}$ is used. The previously described procedure is repeated to identify the model parameters experimentally. Thickness and the gap are found from the optical profilometer scan to be $1.9 \mu \mathrm{m}$, and $1.9 \mu \mathrm{m}$ respectively. Figure 6 shows the natural frequencies as a function of DC voltage for the beam. The effective beam length was determined to be $185 \mu \mathrm{m}$, and the pull-in voltage was estimated to be $14 \mathrm{~V}$ at the zero frequency.

\section{Closed Loop Response}

In the previous section, open loop system parameters were identified and the model dynamic responses were verified through testing. In this section, the closed loop static and dynamic responses are obtained from numerical simulations and are compared with experimental results.

\subsection{Simulation Results}

The closed loop system represented by Equation 5 can show bi-stable behavior as well as single stable behavior [21] by tuning controller gain $G$, and amplification factor $\psi$. A single stable actuator is preferred for position tracking to allow continuous tracking as opposed to the jumps experienced between stable positions occurring in bistable systems. So these parameters are 


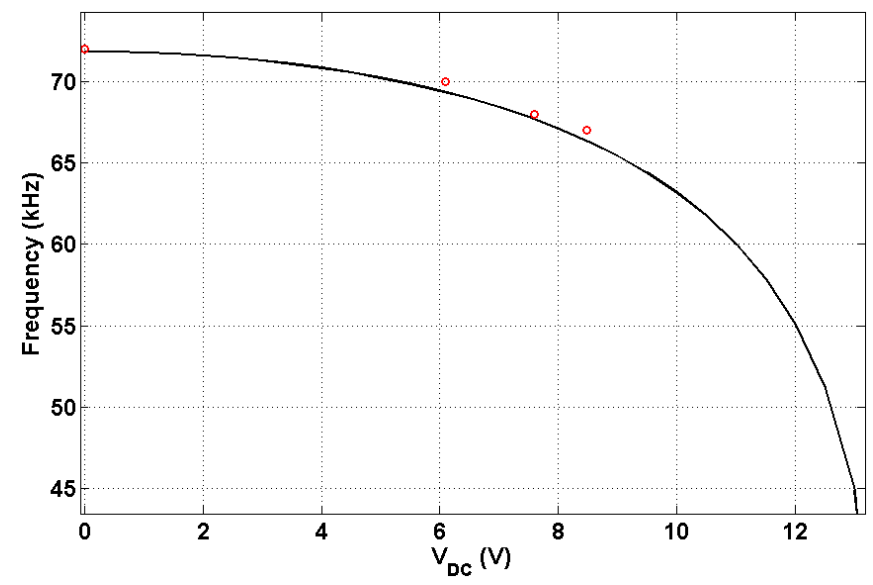

Figure 6: Natural frequency as a function of DC voltage for beam with dimensions of $L=185 \mu \mathrm{m}, b=10 \mu \mathrm{m}, h=1.9 \mu \mathrm{m}$, and $d=1.9 \mu \mathrm{m}$ :Simulations, o experimental results.

chosen as listed in Table 1 to make the system single stable. Figure 7 shows the static response of the system for $G=2, \Psi=1 \mathrm{~V}$ found by setting the time derivatives equal to zero in Equations 5 and solving numerically.

To examine the stability of the equilibrium points, the system equations are linearized around the points and the eigenvalues are found. Table 2 lists the eigenvalues at the equilibrium points corresponding to DC voltages $6 \mathrm{~V}, 8 \mathrm{~V}$, and $12 \mathrm{~V}$. Table 2 results indicate that the equilibrium points along the solid line are stable equilibrium points and the points on the dashed line are the saddle points. It also shows feasible static actuation up to $85 \%$ of the initial gap at $V_{D C}=12 \mathrm{~V}$.

Table 2: Eigenvalues for the equilibrium points at different DC voltages.

\begin{tabular}{|c|c|c|c|c|}
\hline$V_{D C}$ & Point 1 & Eigenvalues 1 & Point 2 & Eigenvalues 2 \\
\hline \hline 6 & 0.203 & $-0.274 \pm 3.06 \mathrm{i}$ & 0.918 & $25,-26$ \\
\hline 8 & 0.57 & $-0.274 \pm 2.716 \mathrm{i}$ & 0.929 & $29.8,-30$ \\
\hline 12 & 0.85 & $-0.274 \pm 13.854 \mathrm{i}$ & 0.945 & $38.573,-39.121$ \\
\hline
\end{tabular}

Dynamic response of the closed loop system is also obtained by converting Equation 5 to state space equations and solving the resulting two ordinary differential equations numerically [21]. Figure 8 shows the simulated steady state dynamic response of the beam excited at $V_{D C}=5 \mathrm{~V}, V_{A C}=3 \mathrm{~V}$, and at a frequency of $10 \mathrm{kHz}$. The input voltage is shown in part (d) of the figure, part

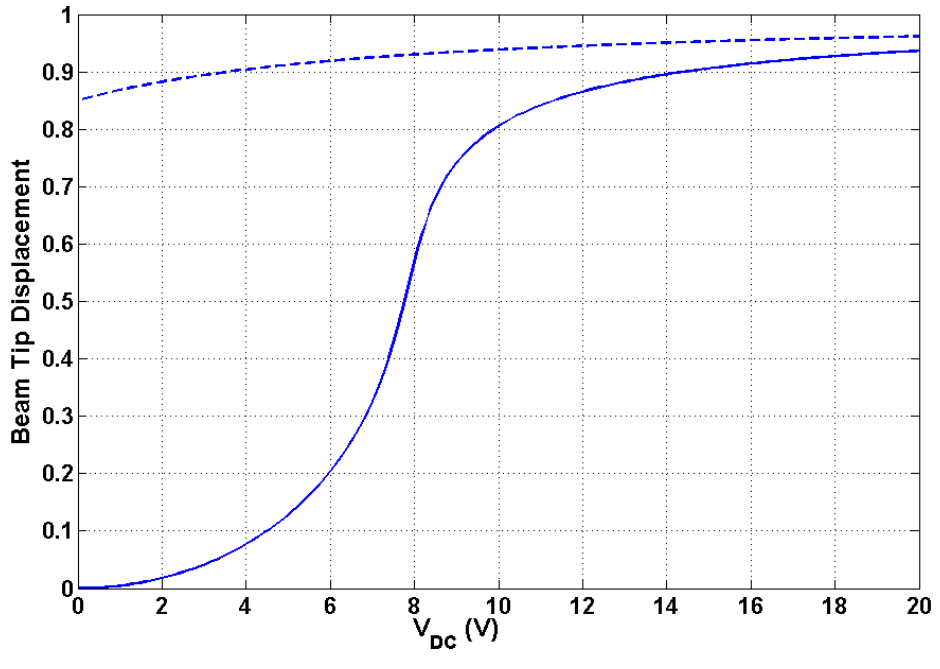

Figure 7: Static response of the closed loop single-stable system. Beam dimensions are $L=185 \mu \mathrm{m}, b=20 \mu \mathrm{m}, h=1.9 \mu \mathrm{m}$, and $d=1.9 \mu \mathrm{m}$ :stable equilibrium points, -- unstable equilibrium points.

(a) shows that the maximum velocity reaches $50 \mathrm{~mm} / \mathrm{s}$, and part (b) shows that the deflection of the beam reaches $1.14 \mu \mathrm{m}(0.57$ of the initial gap) that corresponds the beam tip deflection at the input voltage peak, $V_{D C}=8 \mathrm{~V}$ (Table 2). It is then inferred that the response is forced to maintain the same frequency as the input and to arrive at the same displacement obtained under static (DC) load. This result also indicates that the closed loop actuator can be successful in reaching a desired location in the gap, without going to pull-in. It is also noted that controller output voltage $V_{c}$ is less than $1.6 \mathrm{~V}$ as shown in part (c) of Figure 8.

\subsection{Experimental Results}

The experimental setup for the extended stability actuator consists of a micro-cantilever beam electrostatic actuator, a laser vibrometer, an analog controller circuit, a function generator and a digital oscilloscope. The schematic of the system is shown in Figure 9.The cantilever beam is made of polysilicon and has been fabricated using the PolyMUMPS process. The beam tip velocity is measured by the vibrometer and the corresponding analogue signal is fed to the controller along with the input voltage from the function generator. The controller integrates the signal to find the beam tip displacement, and based on that, it generates a voltage that is subtracted from the input voltage and is multiplied by the gain. The net actuation voltage of $G\left(V_{i n}-V_{c}\right)$ is then fed back to the electrostatic actuator to drive the beam.

A preliminary test has been performed on the closed loop system, that proves we can close the loop and prevent the beam from experiencing pull-in. Figure 10 shows the beam tip velocity recorded by the laser vibrometer. The beam actuator is excited at $V_{D C}=5 \mathrm{~V}, V_{A C}=3 \mathrm{~V}$, and frequency of $\omega=10 \mathrm{kHz}$. Pro- 

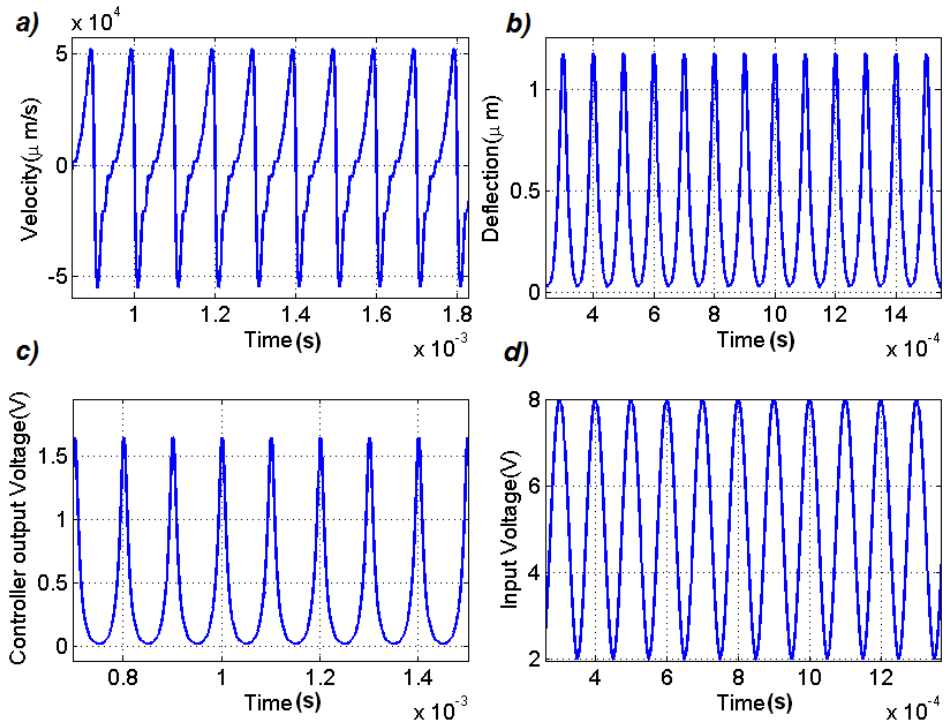

Figure 8: Dynamic response simulation results at $V_{D C}=5 \mathrm{~V}, V_{A C}=3 \mathrm{~V}$, and frequency $\omega=10 \mathrm{kHz}$. Beam dimensions are $L=185 \mu \mathrm{m}, b=$ $20 \mu m, h=1.9 \mu m$, and $d=1.9 \mu m$. a) Beam tip velocity, b) Beam tip deflection, c) Controller voltage $V_{c}$, d) Input voltage $V_{i n}$.

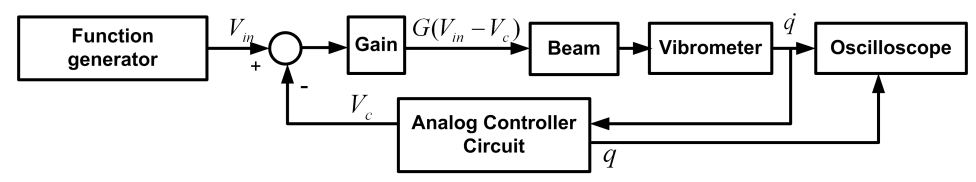

Figure 9: Schematic of the system setup.

viding that the controller did not work, the applied voltage to the actuator would be $G\left(V_{D C}+\frac{V_{A C}}{\sqrt{2}}\right)=2\left(5+\frac{3}{\sqrt{2}}\right)=14.24 \mathrm{~V}$, larger than the $14 \mathrm{~V}$ pull-in voltage, in which case the beam would collapse and would not oscillate. Reaching the maximum velocity of $10 \mathrm{~mm} / \mathrm{s}$ in the closed loop response shows that the controller is working and it is preventing the beam from experiencing pullin. The closed loop velocity response frequency is $10 \mathrm{kHz}$, equal to the input excitation frequency of $10 \mathrm{kHz}$ as expected. However, there exists a $30 \mathrm{~Hz}$ noise produced by a vacuum pump for holding the probe station that is shown in Figure 10 modulated on the $10 \mathrm{kHz}$ response.

A high pass filter is then designed in the analogue controller circuit to filter the $30 \mathrm{~Hz}$ noise. Figure 11 shows dynamic response of the closed loop system to a sinusoidal excitation at $V_{D C}=1.96 \mathrm{~V}, V_{A C}=2 \mathrm{~V}$ after adding the high pass filter. The beam displacement reaches $15 \%$ of the gap and the beam velocity is as large as $13 \mathrm{~mm} / \mathrm{s}$. There is a very close agreement between the beam tip velocity in the experiments and simulations in part a) of Figure 11. The regulated voltage applied to the

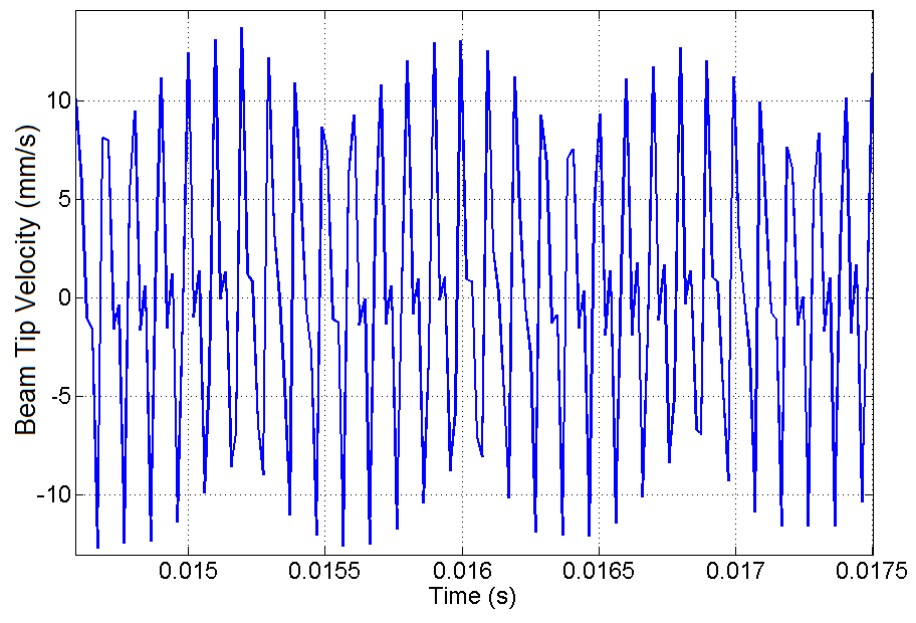

Figure 10: Experimental closed loop response of the electrostatic beam actuator excited at $V_{D C}=5 \mathrm{~V}, V_{A C}=3 \mathrm{~V}$, and frequency $\omega=10 \mathrm{kHz}$. Beam dimensions: $L=185 \mu \mathrm{m}, b=20 \mu \mathrm{m}, h=1.9 \mu \mathrm{m}$, and $d=1.9 \mu \mathrm{m}$.
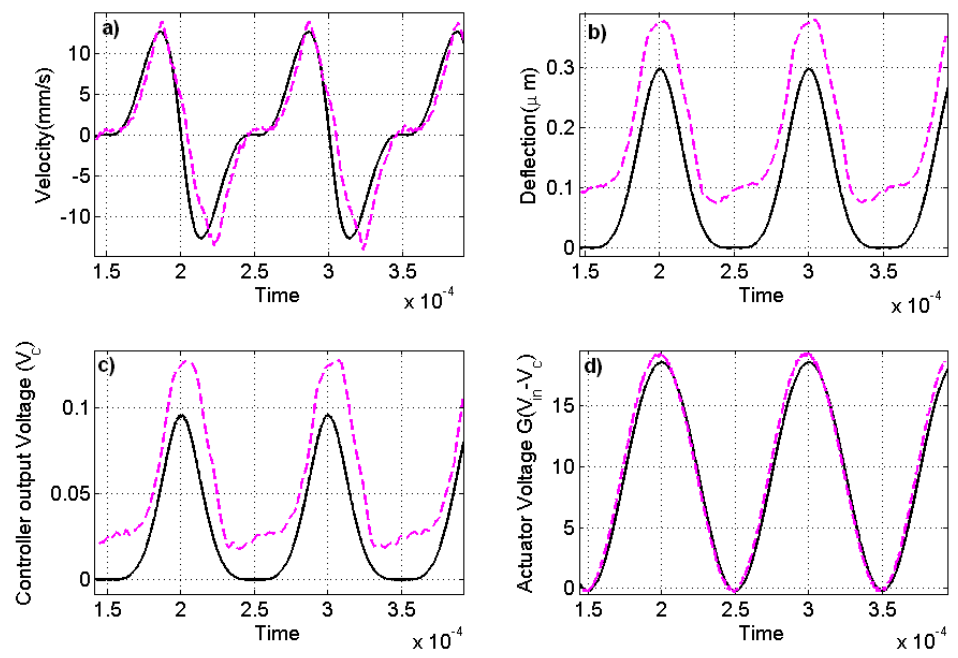

Figure 11: Dynamic response of the closed loop system to $V_{D C}=1.96 \mathrm{~V}$, $V_{A C}=2 \mathrm{~V}, \omega=10 \mathrm{kHz}$. Closed loop circuit parameters are $\Psi=0.45$, $G=4.8$. Beam dimensions are $L=130 \mu m, b=20 \mu m, h=1.9 \mu m, d=$ $1.7 \mu \mathrm{m}$. a) Beam tip velocity, b) Beam tip displacement, c)Controller output voltage, $V_{c}$, d)Regulated voltage applied to the beam $G\left(V_{i n}-V_{c}\right)$ : - Simulations, - - experimental results.

beam in part d) is also very close to what was predicted and is changing between 0 to $20 \mathrm{~V}$. There are slight differences between the simulations and experiments for deflection and the controller output voltage in part b) and c) of Figure 11, which relates to the DC shifting added to the analog integrator to stabilize its function. The overall qualitative and quantitative agreements between simulations and experiments proves that the closed loop system 
model not only tracks desired position but also predicts the system behavior with good accuracy. The future step in our experimental validation is reaching high displacements.

\section{Conclusion}

An analog voltage regulator is developed for an electrostatic actuator. The regulator controls the voltage difference between the two electrodes to balance electrostatic and mechanical forces and to prevent pull-in. The feedback system enables position tracking as well as actuation range extension. The controller was successfully implemented on an analog circuit and integrated with the electrostatic actuator.

This study includes both modeling and testing of the closed loop control system. Model parameters are identified from open loop experiments. The open loop experiments also validated the use of the one mode model presented. In addition, static and dynamic numerical simulations of the closed loop system are demonstrated. Static response of the closed loop system reveals that the actuator can reach up to $85 \%$ of the initial gap. Dynamic response of the closed loop system is also presented and compared with experimental results. Experiments show the effectiveness of the controller in preventing pull-in when a voltage above the pull-in voltage is applied to the feedback system. We have also shown that the closed loop system model is capable of predicting the system behavior with good accuracy. Future work will include experiments with extended deflection range.

\section{Acknowledgment}

This research was financially supported by the Natural Sciences and Engineering Research Council of Canada (NSERC) and the Canadian Foundation for Innovation(CFI). The authors would like to acknowledge the products and services provided by CMC Microsystems (www.cmc.ca) for fabrication using PolyMUMPS technology. The authors would also like to appreciate Prof. Arsen Hajian for kindly providing testing facilities, and Arend von der Lieth for helpful training on the PolyTech vibrometer.

\section{REFERENCES}

[1] H. Xie, Y. Pan, and G.K. Fedder. Endoscopic optical coherence tomographic imaging with a CMOS-MEMS micromirror. Sensors \& Actuators: A. Physical, 103(12):237-241, 2003.

[2] P.H. Tran, D.S. Mukai, M. Brenner, and Z. Chen. In vivo endoscopic optical coherence tomography by use of a rotational microelectromechanical system probe. Optics letters, 29(11):1236-1238, 2004.

[3] JM Zara and SW Smith. Optical scanner using a MEMS actuator. Sensors \& Actuators: A. Physical, 102(1-2):176184, 2002.

[4] D.H. Kim, B. Kim, and H. Kang. Development of a piezoelectric polymer-based sensorized microgripper for microassembly and micromanipulation. Microsystem technologies, 10(4):275-280, 2004.

[5] T. Tanikawa, T. Arai, and T. Masuda. Development of micro manipulation system with two-finger micro hand. In Intelligent Robots and Systems' 96, IROS 96, Proceedings of the 1996 IEEE/RSJ International Conference on, volume 2, 1996.

[6] B. Solano and D. Wood. Design and testing of a polymeric microgripper for cell manipulation. Microelectronic Engineering, 84(5-8):1219-1222, 2007.

[7] K. Kim, X. Liu, Y. Zhang, and Y. Sun. Nanonewton forcecontrolled manipulation of biological cells. Journal of Micromechanics and Microengineering, 18:055013, 2008.

[8] L.R. Carley, J.A. Bain, G.K. Fedder, D.W. Greve, D.F. Guillou, M.S.C. Lu, T. Mukherjee, S. Santhanam, L. Abelmann, and S. Min. Single-chip computers with microelectromechanical systems-based magnetic memory (invited). Journal of Applied Physics, 87:6680, 2000.

[9] DJ Bell, TJ Lu, NA Fleck, and SM Spearing. MEMS actuators and sensors: observations on their performance and selection for purpose. Journal of Micromechanics and $\mathrm{Mi}$ croengineering, 15:S153-S164, 2005.

[10] ES Hung and SD Senturia. Extending the travel range of analog-tuned electrostatic actuators. Microelectromechanical Systems, Journal of, 8(4):497-505, 1999.

[11] S.D. Senturia. Microsystems Design. Kluwer Academic Publishers, Boston, 2001.

[12] J.I. Seeger and B.E. Boser. Dynamics and control of parallel-plate actuators beyond the electrostatic instability. In Transducers, volume 99, pages 474-477. Citeseer, 1999.

[13] P. B. Chu and K. S.J. Pister. Analysis of closed-loop control of parallel-plate electrostatic microgrippers. In Proceedings - IEEE International Conference on Robotics and Automation, volume no. pt 1, pages 820-825, 1994.

[14] M. S.-C. Lu and G. K. Fedder. Position control of parallelplate microactuators for probe-based data storage. Journal of Microelectromechanical Systems, 13(5):759-769, Oct. 2004.

[15] L.A. Rocha, E. Cretu, and R.F. Wolffenbuttel. Using dynamic voltage drive in a parallel-plate electrostatic actuator for full-gap travel range and positioning. Journal of Microelectromechanical Systems, 15(1):69-83, 2006.

[16] M. Vagia, G. Nikolakopoulos, and A. Tzes. Intelligent robust controller design for a micro-actuator. Journal of Intelligent and Robotic Systems, 47(4):299-315, 2006.

[17] B. Borovic, AQ Liu, D. Popa, H. Cai, and FL Lewis. Openloop versus closed-loop control of MEMS devices: choices and issues. Journal of Micromechanics and Microengineer- 
ing, 15:1917, 2005.

[18] S. Towfighian, G. R. Heppler, and E. M. Abdel-Rahman. Analysis of a chaotic electrostatic micro-oscillator. Journal of Computational and Nonlinear Dynamics, In press, Accepted in Feb. 2010.

[19] S. Liu, A. Davidson, and Q. Lin. Simulation studies on nonlinear dynamics and chaos in a MEMS cantilever control system. Journal of Micromechanics and Microengineering, 14(7):1064-1073, 2004.

[20] A. H. Nayfeh, M. L. Younis, and E. M. Abdel-Rahman. Reduced-order models for MEMS applications. Nonlinear Dynamics, 41(1-3):211-236, August 2005.

[21] S. Towfighian, E. M. Abdel-Rahman, and G. R. Heppler. Static and dynamic analysis of a bistable micro-actuator. In Proceedings of ASME International Mechanical Engineering Congress and Exposition, pages 1-11, Boston, MA, USA, 2008. 